\title{
Approximation for Certain Stancu Type Summation Integral Operator
}

\author{
Prerna Maheshwari*
}

Department of Mathematics, SRM University Delhi-NCR Campus, Modinagar (UP), India

Received 30 June 2017; Accepted (in revised version) 28 September 2017

\begin{abstract}
In the present paper, we consider Stancu type generalization of the summation integral type operators discussed in [15]. We apply hypergeometric series for obtaining moments of these operators. We also discuss about asymptotic formula and error estimation in terms of modules of continuity.
\end{abstract}

Key Words: Linear positive operators, hypergeometric series, modulus of continuity.

AMS Subject Classifications: 41A25, 41A28

\section{Introduction}

H. M. Srivastava and V. Gupta [15], proposed a certain family of linear positive operators defined as

$$
G_{n}(f, x)=n \sum_{k=1}^{\infty} p_{n, k}(x, c) \int_{0}^{\infty} p_{n+c, k-1}(t) f(t) d t+p_{n, 0}(x, c) f(0),
$$

$x \in[0, \infty)$, where

$$
p_{n, k}(x, c)=(-1)^{k} \frac{x^{k}}{k !} \phi_{n, c}^{(k)}(x)
$$

and

- for $c=0, \phi_{n}(x)=e^{-n x}$, we obtain Phillips operators,

- for $c \in N, \phi_{n}(x)=(1+c x)^{\frac{-n}{c}}$, we get the discretely defined Baskakov-Durrmeyer operators.

The sequence $\left\{\phi_{n}\right\}_{n \in N}$ of the function defined on an interval $[0, b], b>0$ satisfies the following properties for every $n \in N, k \in N_{0}$,

*Corresponding author. Email address: mprerna_anand@yahoo.com (P. Maheshwari) 
1. $\phi_{n, c} \in C^{\infty}([a, b])$,

2. $\phi_{n, c}(0)=1$,

3. $\phi_{n, c}$ is completely monotone i.e., $(-1)^{k} \phi_{n, c}^{(k)} \geq 0$,

4. There exist an integer $c$ such that $\phi_{n, c}^{(k+1)}(x)=-n \phi_{n+c, c}^{(k)}(x), n>\max \{0, c\}$.

Remark 1.1. Functions $\phi_{n, c}$ have many applications in different fields of Science and Mathematics like potential theory, probability theory, Physics and Numerical Analysis. A collection of most interesting properties of such functions can be found in [17].

These operators are also termed as Srivastava-Gupta operators (see [2,10,16]). In [7], authors have considered the Bezier variant of these operators and estimated the rate of convergence for functions of bounded variation. Motivated by the sequence $G_{n}$, Gupta et al. [4] also defined a mixed family of summation integral operators with different weight function. In approximation theory the genuine type of operators are very important, as they are defined implicitly with values of functions at end points of the interval in which the operators are defined. In 1954, Phillips [14] introduced such operators and later Mazhar and Totik [8] discussed these operators in different form.

In $[5,11,12]$ authors have also studied in this direction and discussed different approximation properties of various operators.

Based on two parameters $\alpha, \beta$ and satisfying the condition $0 \leq \alpha \leq \beta$, motivated by the recent work on Stancu type of generalization (see $[1,9,13])$ in the present paper, we consider the Stancu type generalization of operators (1.1) as

$$
G_{n, c}^{\alpha, \beta}(f, x)=n \sum_{v=1}^{\infty} p_{n, v}(x, c) \int_{0}^{\infty} p_{n+c, v-1}(t, c) f\left(\frac{n t+\alpha}{n+\beta}\right) d t+p_{n, 0}(x, c) f\left(\frac{\alpha}{n+\beta}\right),
$$

where $p_{n, v}(x, c)$ is defined above in (1.2). In this paper, we study simultaneous approximation for the case $c=1$ of the operators defined in (1.3) and establish Voronovskaja type asymptotic formula and error estimation. To obtain moments by using hypergeometric series, we use the technique developed by [6].

\section{Alternate forms}

The operators $G_{n, c}^{\alpha, \beta}(f, x)$ for the case $c=1$ can be written as below. For $x \in[0, \infty)$

$$
\begin{aligned}
G_{n, 1}^{\alpha, \beta}(f, x)= & \int_{0}^{\infty} K_{n}(x, t) f\left(\frac{n t+\alpha}{n+\beta}\right) d t, \\
G_{n, 1}^{\alpha, \beta}(f, x)= & \sum_{v=1}^{\infty} p_{n, v}(x) \int_{0}^{\infty} b_{n, v-1}(t) f\left(\frac{n t+\alpha}{n+\beta}\right) d t \\
& +(1+x)^{-n} f\left(\frac{\alpha}{n+\beta}\right), \quad x \in[0, \infty),
\end{aligned}
$$


where the kernel

$$
K_{n}(x, t)=\sum_{v=1}^{\infty} p_{n, v}(x) b_{n, v-1}(t)+(1+x)^{-n} \delta(t)
$$

with $\delta(t)$ is Dirac delta function and $p_{n, v}(x)$ and $b_{n, v}(t)$ are Baskakov and Beta basis functions and are defined as

$$
\begin{aligned}
& p_{n, v}(x)=\left(\begin{array}{c}
n+v-1 \\
v
\end{array}\right) \frac{x^{v}}{(1+x)^{n+v}}=\frac{(n)_{v}}{v !} \frac{x^{v}}{(1+x)^{n+v}} \\
& b_{n, v-1}(t)=\frac{1}{B(n, v)} \frac{t^{v-1}}{(1+t)^{n+v}}=\frac{(n)_{v}}{(v-1) !} \frac{t^{v-1}}{(1+t)^{n+v}}
\end{aligned}
$$

where the Pochhammer symbol $(n)_{v}$ is defined as

$$
(n)_{v}=n(n+1)(n+2)(n+3) \cdots(n+v-1)
$$

and $B(n, v)$ are Beta functions. The operators $(2.1 \mathrm{~b})$ can be written as

$$
\begin{aligned}
G_{n, 1}^{\alpha, \beta}(f, x)= & \sum_{v=1}^{\infty} \frac{(n)_{v}}{v !} \frac{x^{v}}{(1+x)^{n+v}} \int_{0}^{\infty} \frac{(n)_{v}}{(v-1) !} \frac{t^{v-1}}{(1+t)^{n+v}} \\
& \times f\left(\frac{n t+\alpha}{n+\beta}\right) d t+p_{n, 0}(x) f\left(\frac{\alpha}{n+\beta}\right) \\
= & \int_{0}^{\infty} \frac{f\left(\frac{n t+\alpha}{n+\beta}\right) x}{[(1+x)(1+t)]^{n+1}} \sum_{v=1}^{\infty} \frac{(n)_{v}(n)_{v}}{(v-1) ! v !} \\
& \times \frac{(x t)^{v-1}}{[(1+x)(1+t)]^{v-1}} d t+p_{n, 0}(x) f\left(\frac{\alpha}{n+\beta}\right) \\
= & n^{2} \int_{0}^{\infty} \frac{f\left(\frac{n t+\alpha}{n+\beta}\right) x}{[(1+x)(1+t)]^{n+1}} \sum_{v=0}^{\infty} \frac{(n+1)_{v}(n+1)_{v}}{(v) !(2)_{v}} \\
& \times \frac{(x t)^{v}}{[(1+x)(1+t)]^{v}} d t+p_{n, 0}(x) f\left(\frac{\alpha}{n+\beta}\right) .
\end{aligned}
$$

Using the hypergeometric series properties

$$
{ }_{2} F_{1}(a, b, c ; x)=\sum_{v=0}^{\infty} \frac{(a)_{v}(b)_{v}}{(c)_{v} v !} x^{v},
$$

we have

$$
\begin{aligned}
G_{n, 1}^{\alpha, \beta}(f, x)= & n^{2} \int_{0}^{\infty} \frac{f\left(\frac{n t+\alpha}{n+\beta}\right) x}{[(1+x)(1+t)]^{n+1}} \\
& \times{ }_{2} F_{1}\left(n+1, n+1 ; 2 ; \frac{x t}{(1+x)(1+t)}\right) d t+p_{n, 0}(x) f\left(\frac{\alpha}{n+\beta}\right),
\end{aligned}
$$


on applying Pfaff-Kummar transformation

$$
{ }_{2} F_{1}(a, b, c ; x)=(1-x)^{-a}{ }_{2} F_{1}\left(a, c-b, c ; \frac{x}{x-1}\right),
$$

we get

$$
\begin{aligned}
G_{n, 1}^{\alpha, \beta}(f, x)= & n^{2} \int_{0}^{\infty} \frac{f\left(\frac{n t+\alpha}{n+\beta}\right) x}{(1+x+t)^{n+1}} \\
& \times{ }_{2} F_{1}\left(n+1,1-n ; 2 ; \frac{-x t}{1+x+t}\right) d t+p_{n, 0}(x) f\left(\frac{\alpha}{n+\beta}\right) .
\end{aligned}
$$

This is the alternate form of the operators (2.1b) in terms of hypergeometric function.

\section{Auxiliary results}

In this section, we present some lemmas, which will be useful for the proof of main theorem.

Lemma 3.1. For $n>0$ and $r \geq 1$, we have

$$
G_{n, 1}\left(t^{r}, x\right)=\frac{(n-r-1) ! r !}{(n-1) !} n x(1+x)^{r-1}{ }_{2} F_{1}\left(1-n, 1-r ; 2 ; \frac{x}{1+x}\right)
$$

and

$$
G_{n, 1}\left(t^{r}, x\right)=\frac{(n-r-1) !(n-r-1) !}{((n-1) !)^{2}}+r(r-1) \times \frac{(n+r-2) !(n-r+1) !}{((n-1) !)^{2}} x^{r-1}+\mathcal{O}\left(n^{-2}\right) .
$$

Proof. Taking $f(t)=t^{r}$, using the transformation $t=(1+x) z$ and applying Pfaff-Kummar transformation, we get

$$
\begin{aligned}
& G_{n, 1}\left(t^{r}, x\right) \\
= & n^{2} \int_{0}^{\infty} \frac{x(1+x)^{r} z^{r}}{[(1+x)(1+z)]^{n+1}}(1+x) \sum_{v=0}^{\infty} \frac{(n+1)_{v}(1-n)_{v}}{(2)_{v} v !} \times \frac{(-x(1+x) z)^{v}}{[(1+x)(1+z)]^{v}} d z \\
= & n^{2} \sum_{v=0}^{\infty} \frac{(n+1)_{v}(1-n)_{v}}{(2)_{v} v !}(-x)^{v} x(1+x)^{r-n} \int_{0}^{\infty} \frac{z^{r+v}}{(1+z)^{n+v+1}} d z \\
= & n^{2} \sum_{v=0}^{\infty} \frac{(n+1)_{v}(1-n)_{v}}{(2)_{v} v !}(-x)^{v} x(1+x)^{r-n} B(r+v+1, n-r) \\
= & n^{2} \sum_{v=0}^{\infty} \frac{(n+1)_{v}(1-n)_{v}}{(2)_{v} v !}(-x)^{v} x(1+x)^{r-n} \frac{(r+v) !(n-r-1) !}{(n+v) !}
\end{aligned}
$$


using $(n+v) !=n !(n+1)_{v}$, we get

$$
\begin{aligned}
& G_{n, 1}\left(t^{r}, x\right) \\
= & n^{2} \sum_{v=0}^{\infty} \frac{(n+1)_{v}(1-n)_{v}}{(2)_{v} v !}(-x)^{v} x(1+x)^{r-n} \frac{(n-r-1) !(r+1)_{v} r !}{n !(n+1)_{v}} \\
= & n^{2} x(1+x)^{r-n} \frac{r !(n-r-1) !}{n !} \sum_{v=0}^{\infty} \frac{(r+1)_{v}(1-n)_{v}}{(2)_{v} v !}(-x)^{v} \\
= & n^{2} x(1+x)^{r-n} \frac{r !(n-r-1) !}{n !}{ }_{2} F_{1}(1-n, r+1,2 ;-x) .
\end{aligned}
$$

Thus, we complete the proof.

Now using

we have

$$
{ }_{2} F_{1}(a, b, c ; x)=(1-x)^{-a}{ }_{2} F_{1}\left(a, c-b, c ; \frac{x}{x-1}\right)
$$

$$
\begin{aligned}
G_{n, 1}\left(t^{r}, x\right) & =n^{2} x(1+x)^{r-n} \frac{r !(n-r-1) !}{n !}(1+x)^{n-1}{ }_{2} F_{1}\left(1-n, 1-r, 2 ; \frac{-x}{-x-1}\right) \\
& =n x(1+x)^{r-1} \frac{r !(n-r-1) !}{(n-1) !}{ }_{2} F_{1}\left(1-n, 1-r, 2 ; \frac{x}{x+1}\right) .
\end{aligned}
$$

Lemma 3.2. For $0 \leq \alpha \leq \beta$, we have

$$
\begin{aligned}
G_{n, 1}^{\alpha, \beta}\left(t^{r}, x\right)= & x^{r} \frac{n^{r}}{(n+\beta)^{r}} \frac{(n+r-1) !(n-r-1) !}{((n-1) !)^{2}} \\
& +x^{r-1}\left\{r(r-1) \frac{n^{r}}{(n+\beta)^{r}} \frac{(n+r-2) !(n-r-1) !}{((n-1) !)^{2}}\right. \\
& \left.+r \alpha \frac{n^{r-1}}{(n+\beta)^{r}} \frac{(n+r-2) !(n-r) !}{((n-1) !)^{2}}\right\}+x^{r-2}\{r(r-1) \\
& \times(r-2) \alpha \frac{n^{r-1}}{(n+\beta)^{r}} \frac{(n+r-3) !(n-r) !}{((n-1) !)^{2}}+\frac{r(r-1) \alpha^{2}}{2} \\
& \left.\times \frac{n^{r-2}}{(n+\beta)^{r}} \frac{(n+r-3) !(n-r+1) !}{((n-1) !)^{2}}\right\}+\mathcal{O}\left(\frac{1}{n^{2}}\right) .
\end{aligned}
$$

Proof. The relation between the operators (1.1) and the operators (2.2) can be defined as

$$
\begin{aligned}
G_{n, 1}^{\alpha, \beta}\left(t^{r}, x\right)= & \sum_{j=0}^{r}\left(\begin{array}{c}
r \\
j
\end{array}\right) \frac{n^{j} \alpha^{r-j}}{(n+\beta)^{r}} G_{n}\left(t^{j}, x\right) \\
= & \frac{n^{r}}{(n+\beta)^{r}} G_{n}\left(t^{r}, x\right)+r \alpha \frac{n^{r-1}}{(n+\beta)^{r}} G_{n}\left(t^{r-1}, x\right) \\
& +\frac{r(r-1) \alpha^{2}}{2} \frac{n^{r-2}}{(n+\beta)^{r}} G_{n}\left(t^{r-2}, x\right)+\cdots+\frac{\alpha^{r}}{(n+\beta)^{r}} G_{n}(1, x),
\end{aligned}
$$


by applying (3.2), we get the required result.

Lemma 3.3 (see [3]). Let $m \in N \cup\{0\}$ and

$$
U_{n, m}(x)=\sum_{v=0}^{\infty} p_{n, v}(x)\left(\frac{v}{n}-x\right)^{m}
$$

then $U_{n, 0}(x)=1, U_{n, 1}(x)=0$ and there holds the recurrence relation

$$
n U_{n, m+1}(x)=x(1+x)\left[U_{n, m}^{\prime}(x)+m U_{n, m-1}(x)\right]
$$

and $U_{n, m}(x)=\mathcal{O}\left(n^{\frac{-[m+1]}{2}}\right)$, where $[\alpha]$ being the integral part of $\alpha$.

Lemma 3.4. For $m \in N \cup\{0\}$, we define the central moments as

$$
\begin{aligned}
\mu_{n, m}(x) & =G_{n, 1}^{\alpha, \beta}\left((t-x)^{m}, x\right) \\
& =\sum_{v=1}^{\infty} p_{n, v}(x) \int_{0}^{\infty} b_{n, v-1}(t)\left(\frac{n t+\alpha}{n+\beta}-x\right)^{m} d t+\left(\frac{\alpha}{n+\beta}-x\right)^{m} p_{n, 0}(x)
\end{aligned}
$$

for $n>(m+1)$, following recurrence relation holds

$$
\begin{aligned}
& (n-m-1)\left(\frac{n+\beta}{n}\right) \mu_{n, m+1}(x) \\
= & x(1+x)\left[\mu_{n, m}^{(1)}(x)+m \mu_{n, m-1}(x)\right]+m\left(\frac{\alpha}{n+\beta}-x\right)\left[\left(\frac{\alpha}{n+\beta}-x\right)\right. \\
& \left.\times\left(\frac{n+\beta}{n}\right)-1\right] \mu_{n, m-1}(x)+\left[(n x-1)+(n-2 m-1)\left(\frac{n+\beta}{n}\right)\right. \\
& \left.\times\left(\frac{\alpha}{n+\beta}-x\right)+(m+1)\right] \mu_{n, m}(x),
\end{aligned}
$$

here

$$
\mu_{n, 0}(x)=1 \quad \text { and } \quad \mu_{n, 1}(x)=\frac{x(n+\beta(1-n)+\alpha(n-1)}{(n+\beta)(n-1)} .
$$

Consequently for each $x \in[0, \infty)$, we have from above recurrence relation that

$$
\mu_{n, m}(x)=\mathcal{O}\left(n^{-[m+1] / 2}\right) .
$$

Proof. The values of $\mu_{n, 0}(x)$ and $\mu_{n, 1}(x)$ easily follow from the definition of operators (2.1a). To prove the recurrence relation, we apply the following identities

$$
\begin{aligned}
& x(1+x) p_{n, v}^{(1)}(x)=(v-n x) p_{n, v}(x), \\
& t(1+t) b_{n, v}^{(1)}(t)=[v-(n+1) t] b_{n, v}(t),
\end{aligned}
$$


we get

$$
\begin{aligned}
x(1+x) \mu_{n, m}^{(1)}(x)= & \sum_{v=1}^{\infty}(v-n x) p_{n, v}(x) \int_{0}^{\infty} b_{n, v}(t)\left(\frac{n t+\alpha}{n+\beta}-x\right)^{m} d t \\
& -m x(1+x) \mu_{n, m-1}(x)-n x(1+x)^{-n}\left(\frac{\alpha}{n+\beta}-x\right)^{m} .
\end{aligned}
$$

Therefore,

$$
\begin{aligned}
& x(1+x)\left[\mu_{n, m}^{(1)}(x)+m \mu_{n, m-1}(x)\right] \\
= & \sum_{v=1}^{\infty} p_{n, v}(x) \int_{0}^{\infty}(v-n x) b_{n, v-1}(t)\left(\frac{n t+\alpha}{n+\beta}-x\right)^{m} d t-n x(1+x)^{-n}\left(\frac{\alpha}{n+\beta}-x\right)^{m} \\
= & \sum_{v=1}^{\infty} p_{n, v}(x) \int_{0}^{\infty}[\{(v-1)-(n+1) t\}+(n+1) t+(1-n x)] \\
& \times b_{n, v-1}(t)\left(\frac{n t+\alpha}{n+\beta}-x\right)^{m} d t-n x(1+x)^{-n}\left(\frac{\alpha}{n+\beta}-x\right)^{m} \\
= & \sum_{v=1}^{\infty} p_{n, v}(x) \int_{0}^{\infty} t(1+t) b_{n, v-1}^{(1)}(t)\left(\frac{n t+\alpha}{n+\beta}-x\right)^{m} d t+(n+1) \\
& \times \sum_{v=1}^{\infty} p_{n, v}(x) \int_{0}^{\infty} b_{n, v-1}(t)\left(\frac{n t+\alpha}{n+\beta}-x\right)^{m} d t+(1-n x) \sum_{v=1}^{\infty} p_{n, v}(x) \\
& \times \int_{0}^{\infty} b_{n, v-1}(t)\left(\frac{n t+\alpha}{n+\beta}-x\right)^{m} d t-n x(1+x)^{-n}\left(\frac{\alpha}{n+\beta}-x\right)^{m} .
\end{aligned}
$$

On using the identity

$$
t=\frac{n+\beta}{n}\left[\left(\frac{n t+\alpha}{n+\beta}-x\right)-\left(\frac{\alpha}{n+\beta}-x\right)\right]
$$

we get

$$
\begin{aligned}
& x(1+x)\left[\mu_{n, m}^{(1)}(x)+m \mu_{n, m-1}(x)\right] \\
= & \frac{n+\beta}{n} \sum_{v=1}^{\infty} p_{n, v}(x) \int_{0}^{\infty} b_{n, v-1}^{(1)}(t)\left(\frac{n t+\alpha}{n+\beta}-x\right)^{m+1} d t-\frac{n+\beta}{n} \\
& \times\left(\frac{\alpha}{n+\beta}-x\right) \sum_{v=1}^{\infty} p_{n, v}(x) \int_{0}^{\infty} b_{n, v-1}^{(1)}(t)\left(\frac{n t+\alpha}{n+\beta}-x\right)^{m} d t \\
& +\left(\frac{n+\beta}{n}\right)^{2}\left[\sum_{v=1}^{\infty} p_{n, v}(x) \int_{0}^{\infty} b_{n, v-1}^{(1)}(t)\left(\frac{n t+\alpha}{n+\beta}-x\right)^{m+2} d t\right. \\
& +\left(\frac{\alpha}{n+\beta}-x\right)^{2} \sum_{v=1}^{\infty} p_{n, v}(x) \int_{0}^{\infty} b_{n, v-1}^{(1)}(t)\left(\frac{n t+\alpha}{n+\beta}-x\right)^{m} d t
\end{aligned}
$$




$$
\begin{aligned}
& \left.-2\left(\frac{\alpha}{n+\beta}-x\right) \sum_{v=1}^{\infty} p_{n, v}(x) \int_{0}^{\infty} b_{n, v-1}^{(1)}(t)\left(\frac{n t+\alpha}{n+\beta}-x\right)^{m+1} d t\right] \\
& +(n+1)\left(\frac{n+\beta}{n}\right) \sum_{v=1}^{\infty} p_{n, v}(x) \int_{0}^{\infty} b_{n, v-1}(t)\left(\frac{n t+\alpha}{n+\beta}-x\right)^{m+1} d t \\
& -(n+1)\left(\frac{n+\beta}{n}\right)\left(\frac{\alpha}{n+\beta}-x\right) \sum_{v=1}^{\infty} p_{n, v}(x) \int_{0}^{\infty} b_{n, v-1}(t) \\
& \times\left(\frac{n t+\alpha}{n+\beta}-x\right)^{m} d t+(1-n x)\left[\mu_{n, m}(x)-(1+x)^{-n}\right. \\
& \left.\times\left(\frac{\alpha}{n+\beta}-x\right)^{m}\right]-n x(1+x)^{-n}\left(\frac{\alpha}{n+\beta}-x\right)^{m} .
\end{aligned}
$$

Integrating by parts and rearranging the terms, we get

$$
\begin{aligned}
& (n-m-1)\left(\frac{n+\beta}{n}\right) \mu_{n, m+1}(x) \\
= & x(1+x)\left[\mu_{n, m}^{(1)}(x)+m \mu_{n, m-1}(x)\right]+m\left(\frac{\alpha}{n+\beta}-x\right) \times\left[\left(\frac{\alpha}{n+\beta}-x\right)\left(\frac{n+\beta}{n}\right)-1\right] \mu_{n, m-1}(x) \\
& +\left[(n x-1)+(n-2 m-1)\left(\frac{n+\beta}{n}\right)\left(\frac{\alpha}{n+\beta}-x\right)+(m+1)\right] \mu_{n, m}(x),
\end{aligned}
$$

hence the proof is finished.

Lemma 3.5 (see [3]). There exists the polynomials $\phi_{i, j, r}(x)$, independent of $n$ and $v$ such that

$$
x^{r}(1+x)^{r} \frac{d^{r}}{d x^{r}} p_{n, v}(x)=\sum_{\substack{2 i+j \leq r \\ i, j \geq 0}} n^{i}(v-n x)^{j} \phi_{i, j, r}(x) p_{n, v}(x) .
$$

\section{Main results}

In this section, we prove some direct results including asymptotic formula and error estimation.

Definition 4.1. Let $C_{\gamma}[0, \infty)$ be defined as

$$
C_{\gamma}[0, \infty)=\left\{f \in C[0, \infty): f(t)=\mathcal{O}\left(t^{\gamma}\right), \gamma>0\right\}
$$

then the operators $G_{n, 1}^{\alpha, \beta}(f, x)$ are said to be well defined for $f \in C_{\gamma}[0, \infty)$.

Theorem 4.1. Let $f \in C_{\gamma}[0, \infty)$ be bounded on every finite subinterval of $[0, \infty)$, having the derivatives of order $(r+2)$ at fixed $0<x<\infty$. If $f(t)=\mathcal{O}\left(t^{\gamma}\right)$ as $t \rightarrow \infty$, for some $\gamma>0$, then

$$
\begin{aligned}
& \lim _{n \rightarrow \infty} n\left[\left(G_{n, 1}^{\alpha, \beta}\right)^{(r)}(f, x)-f^{(r)}(x)\right] \\
= & r(r-\beta) f^{(r)}(x)+[(2 r+\alpha)+x(1+r-\beta)] f^{(r+1)}(x)+x(1+x) \times f^{(r+2)}(x) .
\end{aligned}
$$


Proof. We have the Taylor's expansion for the function $f$ as

$$
f(t)=\sum_{i=0}^{r+2} \frac{f^{(i)}(x)}{i !}(t-x)^{i}+\epsilon(t, x)(t-x)^{r+2},
$$

where $\epsilon(t, x) \rightarrow 0$ as $t \rightarrow x$ and $\epsilon(t, x)=o(t-x)^{\delta}$ as $t \rightarrow \infty$, for some $\delta>0$. By using Taylor's expansion, we have

$$
\begin{aligned}
& n\left[\left(G_{n, 1}^{\alpha, \beta}\right)^{(r)}(f, x)-f^{(r)}(x)\right] \\
= & n\left[\sum_{i=0}^{r+2} \frac{f^{(i)}(x)}{i !}\left(G_{n, 1}^{\alpha, \beta}\right)^{(r)}\left((t-x)^{i}, x\right)-f^{(r)}(x)\right]+n\left(G_{n, 1}^{\alpha, \beta}\right)^{(r)}\left(\epsilon(t, x)(t-x)^{r+2}, x\right) \\
= & : I_{1}+I_{2} .
\end{aligned}
$$

Using Lemma 3.2, we have

$$
\begin{aligned}
& I_{1}=n \sum_{i=0}^{r+2} \frac{f^{(i)}(x)}{i !} \sum_{j=r}^{i}\left(\begin{array}{l}
i \\
j
\end{array}\right)(-x)^{i-j}\left(G_{n, 1}^{\alpha, \beta}\right)^{(r)}\left(t^{j}, x\right)-n f^{(r)}(x) \\
& =\frac{f^{(r)}(x)}{r !} n\left[\left(G_{n, 1}^{\alpha, \beta}\right)^{(r)}\left(t^{r}, x\right)-r !\right]+\frac{f^{(r+1)}(x)}{(r+1) !} n\{(r+1)(-x) \\
& \left.\times\left(G_{n, 1}^{\alpha, \beta}\right)^{(r)}\left(t^{r}, x\right)+\left(G_{n, 1}^{\alpha, \beta}\right)^{(r)}\left(t^{r+1}, x\right)\right\}+\frac{f^{(r+2)}(x)}{(r+2) !} n \\
& \times\left\{\frac{(r+1)(r+2)}{2} x^{2}\left(G_{n, 1}^{\alpha, \beta}\right)^{(r)}\left(t^{r}, x\right)+(r+2)(-x)\right. \\
& \left.\times\left(G_{n, 1}^{\alpha, \beta}\right)^{(r)}\left(t^{r+1}, x\right)+\left(G_{n, 1}^{\alpha, \beta}\right)^{(r)}\left(t^{r+2}, x\right)\right\} \\
& =n\left[\frac{n^{r}(n+r-1) !(n-r-1) !}{(n+\beta)^{r}((n-1) !)^{2}}-1\right] f^{(r)}(x)+n \frac{f^{(r+1)}(x)}{(r+1) !}[(r+1) \\
& \times(-x) \frac{n^{r}(n+r-1) !(n-r-1) !}{(n+\beta)^{r}((n-1) !)^{2}} r !+n^{r+1} \frac{(n+r) !(n-r-2) !}{(n+\beta)^{r+1}((n-1) !)^{2}} \\
& \times(r+1) ! x+\frac{r(r+1) n^{r+1}(n+r-1) !(n-r-2) !}{(n+\beta)^{r+1}((n-1) !)^{2}} r ! \\
& \left.+(r+1) \alpha \frac{n^{r}(n+r-1) !(n-r-1) !}{(n+\beta)^{r+1}((n-1) !)^{2}} r !\right] \\
& +n \frac{f^{(r+2)}(x)}{(r+2) !}\left\{\frac{(r+2)(r+1)}{2} x^{2} \frac{n^{r}(n+r-1) !(n-r-1) !}{(n+\beta)^{r}((n-1) !)^{2}} r !-(r+2) x\right. \\
& \times\left(\frac{n^{r+1}(n+r) !(n-r-2) !}{(n+\beta)^{r+1}((n-1) !)^{2}}(r+1) ! x+r(r+1) n^{r+1}\right. \\
& \left.\times \frac{(n+r-1) !(n-r-2) !}{(n+\beta)^{r+1}((n-1) !)^{2}} r !+(r+1) \alpha \frac{n^{r}(n+r-1) !(n-r-1) !}{(n+\beta)^{r+1}((n-1) !)^{2}} \times r !\right)
\end{aligned}
$$




$$
\begin{aligned}
& +n^{r+2} \frac{(n+r+1) !(n-r-3) !}{(n+\beta)^{r+2}((n-1) !)^{2}} \frac{(r+2) !}{2} x^{2}+(r+1)(r+2) \\
& \times n^{r+2} \frac{(n+r) !(n-r-3) !}{(n+\beta)^{r+2}((n-1) !)^{2}}(r+1) ! x+(r+2) \alpha n^{r+1} \\
& \times \frac{(n+r) !(n-r-2) !}{(n+\beta)^{r+2}((n-1) !)^{2}}(r+1) ! x+r(r+1)(r+2) \alpha n^{r+1} \\
& \times \frac{(n+r-1) !(n-r-2) !}{(n+\beta)^{r+2}((n-1) !)^{2}} r !+(r+1)(r+2) \alpha^{2} n^{r} \\
& \left.\times \frac{(n+r-1) !(n-r-1) !}{2(n+\beta)^{r+2}((n-1) !)^{2}} r !\right\}+\mathcal{O}\left(n^{-2}\right) .
\end{aligned}
$$

In the above expression $r(r-\beta),(2 r+\alpha)+x(1+r-\beta)$ and $x(1+x)$ are the coefficients of $f^{(r)}(x), f^{(r+1)}(x)$ and $f^{(r+2)}(x)$ respectively, which easily follow by using induction hypothesis on $r$ and then taking limit as $n \rightarrow \infty$. Therefore in order to complete the proof of above theorem, it is sufficient to show that $I_{2} \rightarrow 0$ as $n \rightarrow \infty$. For this using Lemma 3.5, we have

$$
\begin{aligned}
\left|I_{2}\right| \leq & \sum_{\substack{2 i+j \leq r \\
i, j \geq 0}} \frac{n^{i}\left|\phi_{i, j, r}(x)\right|}{x^{r}(1+x)^{r}} \sum_{v=1}^{\infty}|v-n x|^{j} p_{n, v}(x) \int_{0}^{\infty} b_{n, v-1}(t)|\epsilon(t, x)| \\
& \times\left|\frac{n t+\alpha}{n+\beta}-x\right|^{r+2} d t+(-1)^{r} \frac{(n+r-1) !}{n !}|\epsilon(0, x)|\left|\frac{\alpha}{n+\beta}-x\right|^{r+2} \\
= & : I_{3}+I_{4},
\end{aligned}
$$

as $\epsilon(t, x) \rightarrow 0$ when $t \rightarrow x$, hence for a given $\epsilon>0$, there exists a positive number $\delta:|\epsilon(t, x)|<$ $\epsilon$ whenever $|t-x|<\delta$. If $\lambda$ is any integer $\geq \max \{\lambda, r+2\}$ then for a constant $M>0$, which does not depend on $t$, we have

$$
|\epsilon(t, x)|\left|\frac{n t+\alpha}{n+\beta}-x\right|^{r+2} \leq M\left|\frac{n t+\alpha}{n+\beta}-x\right|^{\lambda},
$$

where $|t-x| \geq \delta$. Hence

$$
\begin{aligned}
\left|I_{3}\right|= & M_{1} \sum_{\substack{2 i+j \leq r \\
i, j \geq 0}} n^{i+1} \sum_{v=1}^{\infty}|v-n x|^{j} p_{n, v}(x)\left\{\int_{|t-x|<\delta} \epsilon b_{n, v-1}(t)\right. \\
& \left.\times\left|\frac{n t+\alpha}{n+\beta}-x\right|^{r+2} d t+\int_{|t-x| \geq \delta} M b_{n, v-1}(t)\left|\frac{\ell}{n t+\alpha} n+\beta-x\right|^{\lambda} d t\right\} \\
= & : I_{5}+I_{6},
\end{aligned}
$$

where

$$
M_{1}=\sum_{\substack{2 i+j \leq r \\ i, j \geq 0}} \frac{\left|\phi_{i, j, r}(x)\right|}{x^{r}(1+x)^{r}} .
$$


Using Schwarz's inequality, we have

$$
\begin{aligned}
\left|I_{5}\right| \leq & \in M_{1} \sum_{\substack{2 i+j \leq r \\
i, j \geq 0}} n^{i+1} \sum_{v=1}^{\infty}|v-n x|^{j} p_{n, v}(x)\left(\int_{0}^{\infty} b_{n, v-1}(t) d t\right)^{1 / 2} \\
& \times\left(\int_{0}^{\infty} b_{n, v-1}(t)\left(\frac{n t+\alpha}{n+\beta}-x\right)^{2 r+4} d t\right)^{1 / 2} \\
\leq & \epsilon M_{1} \sum_{\substack{2 i+j \leq r \\
i, j \geq 0}} n^{i+1}\left(\sum_{v=1}^{\infty}(v-n x)^{2 j} p_{n, v}(x)\right)^{1 / 2} \\
& \times\left(\sum_{v=1}^{\infty} p_{n, v}(x) \int_{0}^{\infty} b_{n, v-1}(t)\left(\frac{n t+\alpha}{n+\beta}-x\right)^{2 r+4} d t\right)^{1 / 2}
\end{aligned}
$$

On using Lemma 3.3 and Lemma 3.4, we have

$$
\left|I_{5}\right| \leq \epsilon M_{1} \sum_{\substack{2 i+j \leq r \\ i, j \geq 0}} n^{i+1} \mathcal{O}\left(n^{j / 2}\right) \cdot \mathcal{O}\left(n^{-(r+2) / 2}\right) \leq \epsilon \mathcal{O}(1)
$$

and as $\epsilon$ is arbitrary, it implies that $I_{5}=o(1)$.

Again applying Schwarz's inequality for summation and integration and then applying Lemma 3.3 and Lemma 3.4, we have

$$
\begin{aligned}
\left|I_{6}\right| \leq & M_{2} \sum_{\substack{2 i+j \leq r \\
i, j \geq 0}} n^{i+1} \sum_{v=1}^{\infty}|v-n x|^{j} p_{n, v}(x) \int_{|t-x| \geq \delta} b_{n, v-1}(t) \times\left|\frac{n t+\alpha}{n+\beta}-x\right|^{\lambda} d t \\
\leq & M_{2} \sum_{\substack{2 i+j \leq r \\
i, j \geq 0}} n^{i+1}\left(\sum_{v=1}^{\infty} p_{n, v}(x)(v-n x)^{2 j}\right)^{1 / 2} \\
& \times\left(\sum_{v=1}^{\infty} p_{n, v}(x) \int_{0}^{\infty} b_{n, v-1}(t)\left(\frac{n t+\alpha}{n+\beta}-x\right)^{2 \lambda} d t\right)^{1 / 2} \\
= & \sum_{\substack{2 i+j \leq r \\
i, j \geq 0}} n^{i+1} \cdot \mathcal{O}\left(n^{j / 2}\right) \cdot \mathcal{O}\left(n^{-\lambda / 2}\right) \\
= & \mathcal{O}\left(n^{(r+2-\lambda) / 2}\right)=o(1) .
\end{aligned}
$$

Hence $I_{3} \rightarrow 0$ as $n \rightarrow \infty$. Since it is clear that $I_{4} \rightarrow 0$ as $n \rightarrow \infty$, we get $I_{2}=o(1)$. Combining the estimates of $I_{1}$ and $I_{2}$, we get the required result.

Hence the proof of the theorem is completed. 
Theorem 4.2. For $f \in C_{\gamma}[0, \infty)$, for some $\gamma>0$ and $r \leq k \leq r+2$. If $f^{(k)}$ exists and is continuous on $(a-\eta, b+\eta) \subset(0, \infty)$, where $\eta>0$, then for sufficiently large $n$

$$
\begin{aligned}
& \left\|\left(G_{n, 1}^{\alpha, \beta}\right)^{(r)}(f, x)-f^{(r)}(x)\right\|_{C[a, b]} \\
\leq & M_{1} \frac{1}{n} \sum_{i=r}^{k}\left\|f^{(i)}\right\|_{C[a, b]}+M_{2} n^{-1 / 2} \omega\left(f^{(k)}, n^{-1 / 2}\right)+\mathcal{O}\left(1 / n^{2}\right),
\end{aligned}
$$

where $M_{1}$ and $M_{2}$ are constants independent of $f$ and $n, \omega(f, \delta)$ is the modulus of continuity of $f$ on $(a-\eta, b+\eta)$ and $\|\cdot\|_{C[a, b]}$ denotes the sup-norm on the interval $[a, b]$.

Proof. Using Taylor's expansion on function $f$, we have

$$
f(t)=\sum_{i=0}^{k} \frac{f^{(i)}(x)}{i !}(t-x)^{i}+\frac{f^{(k)}(\theta)-f^{(k)}(x)}{k !}(t-x)^{k} \xi(t)+h(t, x)(1-\xi(t)),
$$

where $\theta$ lies between $t$ and $x$, and $\xi(t)$ is the characteristic function on the interval $(a-$ $\eta, b+\eta)$. Therefore

$$
\begin{aligned}
& \left(G_{n, 1}^{\alpha, \beta}\right)^{(r)}(f, x)-f^{(r)}(x) \\
= & \left\{\sum_{i=0}^{k} \frac{f^{(i)}(x)}{i !}\left(G_{n, 1}^{\alpha, \beta}\right)^{(r)}\left((t-x)^{i}, x\right)-f^{(r)}(x)\right\} \\
& +\left(G_{n, 1}^{\alpha, \beta}\right)^{(r)}\left(\frac{f^{(k)}(\eta)-f^{(k)}(x)}{k !}(t-x)^{k} \xi(t), x\right) \\
& +\left(G_{n, 1}^{\alpha, \beta}\right)^{(r)}(h(t, x)(1-\xi(t)), x) \\
= & : L_{1}+L_{2}+L_{3} .
\end{aligned}
$$

Using Lemma 3.2, we have

$$
\begin{aligned}
L_{1}= & \sum_{i=0}^{k} \frac{f^{(i)}(x)}{i !} \sum_{j=0}^{i}\left(\begin{array}{c}
i \\
j
\end{array}\right)(-x)^{i-j} \frac{d^{r}}{d x^{r}}\left[\frac{n^{j} \Gamma(n+j) \Gamma(n-j)}{(n+\beta)^{j}(\Gamma n)^{2}} x^{j}\right. \\
& +\left(j(j-1) n^{j} \frac{\Gamma(n+j-1) \Gamma(n-j)}{(n+\beta)^{j}(\Gamma n)^{2}}+j \alpha n^{j-1}\right. \\
& \left.\times \frac{\Gamma(n+j-1) \Gamma(n-j+1)}{(n+\beta)^{j}(\Gamma n)^{2}}\right) x^{j-1}+\left(j(j-1)(j-2) \alpha n^{j-1}\right. \\
& \times \alpha \frac{\Gamma(n+j-2) \Gamma(n-j+1)}{(n+\beta)^{j}(\Gamma n)^{2}}+j(j-1) \alpha^{2} n^{j-2} \\
& \left.\left.\times \frac{\Gamma(n+j-2) \Gamma(n-j+2)}{2(n+\beta)^{j}(\Gamma n)^{2}}\right) x^{j-2}+\mathcal{O}\left(\frac{1}{n^{2}}\right)\right]-f^{(r)}(x) .
\end{aligned}
$$


Hence

$$
\left\|L_{1}\right\|_{C[a, b]} \leq M_{1} \cdot \frac{1}{n} \sum_{i=r}^{k}\left\|f^{(i)}\right\|_{C[a, b]}+\mathcal{O}\left(\frac{1}{n^{2}}\right) \quad \text { on }[a, b] .
$$

To estimate $L_{2}$, we follow

$$
\begin{aligned}
\left|L_{2}\right| \leq & \int_{0}^{\infty}\left|K_{n}^{(r)}(x, t)\right|\left\{\left|\frac{f^{(k)}(\theta)-f^{(k)}(x)}{k !}\right|\left|\frac{n t+\alpha}{n+\beta}-x\right|^{k} \xi(t)\right\} d t \\
\leq & \frac{\omega\left(f^{(k)}, \delta\right)}{k !} \int_{0}^{\infty}\left|K_{n}^{(r)}(x, t)\right|\left(1+\frac{\left|\frac{n t+\alpha}{n+\beta}-x\right|}{\delta}\right)\left|\frac{n t+\alpha}{n+\beta}-x\right|^{k} d t \\
\leq & \frac{\omega\left(f^{(k)}, \delta\right)}{k !}\left[\sum _ { v = 1 } ^ { \infty } | p _ { n , v } ^ { ( r ) } ( x ) | \int _ { 0 } ^ { \infty } b _ { n , v - 1 } ( t ) \left(\left|\frac{n t+\alpha}{n+\beta}-x\right|^{k}\right.\right. \\
& \left.+\frac{1}{\delta}\left|\frac{n t+\alpha}{n+\beta}-x\right|^{k+1}\right) d t+\frac{\Gamma(n+r)}{\Gamma n}(1+x)^{-(n+r)} \\
& \left.\times\left(\left|\frac{\alpha}{n+\beta}-x\right|^{k}+\frac{1}{\delta}\left|\frac{\alpha}{n+\beta}-x\right|^{k+1}\right)\right] .
\end{aligned}
$$

By applying Schwarz's inequality for summation and integral, we have

$$
\begin{aligned}
& \sum_{v=1}^{\infty}|v-n x|^{j} p_{n, v}(x) \int_{0}^{\infty} b_{n, v-1}(t)\left|\frac{n t+\alpha}{n+\beta}-x\right|^{k} d t \\
\leq & \sum_{v=1}^{\infty} p_{n, v}(x)|v-n x|^{j}\left(\int_{0}^{\infty} b_{n, v-1}(t) d t\right)^{1 / 2} \\
& \times\left(\int_{0}^{\infty} b_{n, v-1}(t)\left(\frac{n t+\alpha}{n+\beta}-x\right)^{2 k} d t\right)^{1 / 2} \\
\leq & \left(\sum_{v=1}^{\infty} p_{n, v}(x)(v-n x)^{2 j}\right)^{1 / 2} \\
& \times\left(\sum_{v=1}^{\infty} p_{n, v}(x) \int_{0}^{\infty} b_{n, v-1}(t)\left(\frac{n t+\alpha}{n+\beta}-x\right)^{2 k} d t\right)^{1 / 2} \\
= & \mathcal{O}\left(n^{j / 2}\right) \times \mathcal{O}\left(n^{-k / 2}\right) \\
= & \mathcal{O}\left(n^{\frac{(j-k)}{2}}\right) \text { on the interval }[a, b] .
\end{aligned}
$$

Therefore by Lemma 3.5 and (4.1), we get

$$
\sum_{v=1}^{\infty}\left|p_{n, v}^{(r)}(x)\right| \int_{0}^{\infty} b_{n, v-1}(t)\left|\frac{n t+\alpha}{n+\beta}-x\right|^{k} d t
$$




$$
\begin{aligned}
\leq & \sum_{v=1}^{\infty} \sum_{\substack{2 i+j \leq r \\
i, j \leq 0}} \frac{n^{i}\left|\phi_{i, j, r}(x)\right|}{x^{r}(1+x)^{r}}|v-n x|^{j} p_{n, v}(x) \int_{0}^{\infty} b_{n, v-1}(t) \times\left|\frac{n t+\alpha}{n+\beta}-x\right|^{k} d t \\
\leq & \left(\sup _{\substack{2 i+j \leq r \\
i, j \leq 0}} \frac{\left|\phi_{i, j, r}(x)\right|}{x^{r}(1+x)^{r}}\right) \sum_{\substack{2 i+j \leq r \\
i, j \geq 0}} n^{i} \\
& \times\left(\sum_{v=1}^{\infty}|v-n x|^{j} p_{n, v}(x) \int_{0}^{\infty} b_{n, v-1}(t)\left|\frac{n t+\alpha}{n+\beta}-x\right|^{k} d t\right) \\
= & M \sum_{\substack{2 i+j \leq r \\
i, j \leq 0}} n^{i} \cdot \mathcal{O}\left(n^{\frac{(j-k)}{2}}\right) \\
= & \mathcal{O}\left(n^{\frac{(r-k)}{2}}\right),
\end{aligned}
$$

on $[a, b]$, where

$$
M=\sup _{\substack{2 i+j \leq r \\ i, j \geq 0}} \sup _{x \in[a, b]} \frac{\left|\phi_{i, j, r}(x)\right|}{x^{r}(1+x)^{r}} .
$$

If we choose $\delta=1 / \sqrt{n}$ and apply (4.2) we have

$$
\begin{aligned}
\left\|L_{2}\right\|_{C[a, b]} & \leq \frac{\omega\left(f^{(k)}, \frac{1}{\sqrt{n}}\right)}{k !}\left[\mathcal{O}\left(n^{(r-k) / 2}\right)+\sqrt{n} \mathcal{O}\left(n^{(r-k-1) / 2}\right)+\mathcal{O}\left(1 / n^{m}\right)\right] \\
& \leq M_{2} n^{-(r-k) / 2} \omega\left(f^{(k)}, 1 \sqrt{n}\right) .
\end{aligned}
$$

Since $t \in[0, \infty) \backslash(a-\eta, b+\eta)$, here we opt $\delta$ such that $|t-x| \geq \delta$ for all $x \in[a, b]$. Hence by Lemma 3.5

$$
\begin{aligned}
\left|L_{3}\right|= & M \sum_{\substack{2 i+j \leq r \\
i, j \geq 0}} n^{i} \sum_{v=1}^{\infty}|v-n x|^{j} p_{n, v}(x) \int_{|t-x| \geq \delta} b_{n, v-1}(t)|h(t, x)| d t \\
& +\frac{(n+r-1) !}{(n-r) !}(1+x)^{-(n+r)}|h(0, x)|
\end{aligned}
$$

for $|t-x| \geq \delta$, we find a constant $K$ such that

$$
|h(t, x)| \leq K\left|\frac{n t+\alpha}{n+\beta}-x\right|^{\beta}
$$

where $\beta \geq\{\gamma, k\}$ is an integer. Therefore by using Schwarz's inequality for summation and integration and using Lemma 3.3 as well as Lemma 3.4, we follow that $L_{3}=\mathcal{O}\left(1 / n^{s}\right)$, for any $s>0$ uniformly on $[a, b]$.

Combining the estimates of $L_{1}, L_{2}$ and $L_{3}$, we get the required result. 


\section{Acknowledgements}

The author would like to thank to the referees for their valuable suggestion in improving the quality of paper. Author is also thankful to National Board of Higher Mathematics, for providing a plate-form for research.

\section{References}

[1] I. Buyukyazici and C. Atakut, On Stancu type generalization of $q$-Baskakov operators, Math. Comput. Model., 52(5-6) (2010), 752-759.

[2] N. Deo, Faster rate of convergence on Srivastava-Gupta operators, Appl. Math. Comput., 218(21) (2012), 10486-10491.

[3] V. Gupta, Error estimates for mixed summation integral type operators, J. Math. Anal. Appl., 313 (2006), 632-641.

[4] V. Gupta, R. N. Mohapatra and Z. Finta, On certain family of mixed summation-integral operators, Math. Comput. Model., 42 (2005), 181-191.

[5] V. Gupta and P. Maheshwari, Bezier variant of a new Durrmeyer type operators, Rivista Di Mate. Della Univ. di Parma, 7(2) (2003), 9-21.

[6] M. Ismail and P. Simeonov, On a family of positive linear operators in: notions of positively and the geometry of polynomials, Trends in Mathematics, (2011), 259-274.

[7] N. Ispir and I. Yuksel, On the Bezier variant of Srivastava-Gupta operators, Appl. Math. E-Notes, 5 (2005), 129-137.

[8] S. M. Majharand and V. Totik, Approximation by modified Szasz operators, Acta. Sci. Math., 49 (1985), 257-269.

[9] P. Maheshwari, Approximation properties of certain $q$-genuine Szasz operators, Complex Analysis and Operator Theory, DOI 10.1007/s11785-016-0538-3 (2016), 1-10.

[10] P. Maheshwari, On modified Srivastava-Gupta operators, Filomat, 29(6) (2015), 1173-1177.

[11] P. Maheshwari, Saturation theorem for the combinations of modified Beta operators in $L_{p}$ spaces, Appl. Math. E-Notes, 7 (2007), 32-37.

[12] P. Maheshwari, An Inverse result in simultaneous approximation of modified Beta operators, Georgian Math. J., 13(2) (2006), 297-306.

[13] P. Maheshwari and D. Sharma, Approximation by $q$-Baskakov-Beta-Stancu operators, Rend. Circ. Mat. Palermo, 61 (2012), 297-305.

[14] R. S. Phillips, An inversion formula for semi-groups of linear operators, Ann. Math., 59(2) (1954), 352-356.

[15] H. M. Srivastava and V. Gupta, A certain family of summation integral type operators, Math. Comput. Model., 37(12-13) (2003), 1307-1315.

[16] D. K. Verma and P. N. Agarwal, Convergence in simultaneous approximation for SrivastavaGupta operators, Math. Sci., 6(22) (2012), doi:10.1186/2251-7456-6-22.

[17] D. V. Widder, The Laplace Transform, Princeton University Press, Princeton 1941. 